\title{
Upregulation of ACE2 and TMPRSS2 by particulate matter and idiopathic pulmonary fibrosis: $A$ potential role in severe COVID-19
}

Hsin-Hsien Li

National Yang-Ming University

Chen-Chi Liu

National Yang-Ming University

Tien-Wei Hsu

National Yang-Ming University

Jiun-Han Lin

National Yang-Ming University

Jyuan-Wei Hsu

National Yang-Ming University

Anna Fen-Yau Li

National Yang-Ming University

Yi-Chen Yeh

National Yang-Ming University

Han-Shui Hsu ( $\square$ hsuhs@vghtpe.gov.tw)

National Yang-Ming University

Shih-Chieh Hung ( $\sim$ hung3340@gmail.com )

China Medical University

\section{Research Article}

Keywords: Air pollution, idiopathic pulmonary fibrosis, COVID-19, ACE2, TMPRSS2

Posted Date: November 20th, 2020

DOl: https://doi.org/10.21203/rs.3.rs-112349/v1

License: (1) (i) This work is licensed under a Creative Commons Attribution 4.0 International License.

Read Full License 


\section{Abstract}

Background: Air pollution and idiopathic pulmonary fibrosis (IPF) cause a poor prognosis after COVID-19 infection, but the underlying mechanisms are not well exploited. Angiotensin-converting enzyme 2 (ACE2) and transmembrane serine protease 2 (TMPRSS2) are the keys to the entry of SARS-CoV-2. We measured their expression levels in lung tissues of control non-IPF and IPF patients, and used murine animal models to study the deterioration of IPF caused by particulate matter (PM) and the molecular pathways involved in the expression of ACE2 and TMPRSS2.

Results: In non-IPF patients, cells expressing ACE2 and TMPRSS2 were limited to human alveolar cells. ACE2 and TMPRSS2 were largely upregulated in IPF patients, and were co-expressed by fibroblast specific protein 1 (FSP-1)+ lung fibroblasts in human pulmonary fibrotic tissue. In animal models, PM exposure increased the severity of bleomycin-induced pulmonary fibrosis. ACE2 and TMPRSS2 were also expressed in FSP-1+ lung fibroblasts in bleomycin-inuced pulmonary fibrosis, and when combined with PM exposure, they were further upregulated. The severity of pulmonary fibrosis and the expression of ACE2 and TMPRSS2 caused by PM exposure were blocked by deletion of KC, a murine homologue of IL8 , or treatment with reparixin, an inhibitor of IL-8 receptors CXCR1/2.

Conclusions: These data suggest that poor prognosis after COVID-19 infection caused by air pollution and IPF is mediated through upregulation of ACE2 and TMPRSS2 in pulmonary fibroblasts, which can be prevented by blocking the IL-8/CXCR1/2 pathway.

\section{Introduction}

In December 2019, an outbreak of a novel severe acute respiratory syndrome coronavirus 2 (SARS-CoV2) began in Wuhan, China. The World Health Organization (WHO) named it the coronavirus disease (COVID-19) and assigned the outbreak as a public health emergency. As of early August 2020, more than 20 million confirmed cases and 700,000 deaths have been reported worldwide. The COVID-19 pandemic is a global health crisis. Since, currently there are no established therapies and vaccines, it is critical to understand the factors mediating and influencing the outcome of the disease.

A significant correlation exists between short-term exposure to higher levels of particulate matter (PM) and COVID-19 infection [1]. Comprehensive time trend data of the atmosphere and coronavirus provide evidence of the probable impacts of air pollution on the rapid spread of SARS-CoV-2 and COVID19 in Milan [2]. In November 2002, during the SARS outbreak in mainland China, a linear relationship was established between the air pollution index (API) and fatality rate [3]. Although there are several influencing factors such as age, regional health system, preventive policies, and intensive care capacity, air pollution is an important factor in the lethality of COVID-19 in northern Italy [4]. Recently, Società Italiana di Medicina Ambientale (SIMA) reported remarkable spread of COVID-19 virus in some areas of northern Italy possibly through air pollution [5]. However, the primary etiologies that air pollution or PM affects the entry or spread of SARS-CoV-2 are mainly unclarified. 
Idiopathic pulmonary fibrosis (IPF) is a chronic, progressive disease characterized by declining lung function, leading to respiratory failure and a poor prognosis [6]. Several pulmonary and extrapulmonary complications are also associated with IPF, including pulmonary hypertension, emphysema, lung cancer, venous thromboembolism, coronary artery disease, and congestive heart failure [7]. Accumulated evidence shows that the major risk factors for severe COVID-19 coexist with IPF, namely age, male gender, smoking history and comorbidities, such as hypertension and diabetes [8]. Patients with interstitail lung disease are at increased risk of death from COVID-19, particularly those with a Forced Vital Capacity (FVC) less than $80 \%$ predicited and obesity [9]. Because the prognosis of patients with IPF when infected with COVID-19 is worse than that of the general population, anti-fibrosis therapy may have potential value in preventing and treating such patients when infected with COVID-19 [10]. However, the underlying moleclar mechanism regarding the poor outcome of IPF patients with COVID-19 infection and the role of antifibrotic therapy in SARS-CoV-2 infection are not well explored.

Angiotensin-converting enzyme 2 (ACE2), a functional receptor in the lung, was found to be a key site for transmission of SARS coronavirus (SARS-CoV) [11]. The spike protein of SARS-CoV attaches the virus to ACE2 and employs the cellular serine protease TMPRSS2 for S protein priming . Similarly, SARSCoV-2 enters the host cell using ACE2 and TMPRSS2, but the pathway is intercepted by a clinically proven protease inhibitor [12].

Studies have shown that air pollution, especially nitrogen dioxide $\left(\mathrm{NO}_{2}\right)$ and particulate matter 2.5 $\left(\mathrm{PM}_{2.5}\right)$, may negatively impact the incidence [13] and results [14], and cause acute exacerbation of IPF [15]. Industrial areas, such as the Lombardy region of northern Italy, are characterized by high levels of air pollution with high prevalence of IPF [13], and have been severely affected by the COVID-19 pandemic with high mortality rates [4]. Although COVID-19 spreads primarily through aerosols, droplets, and direct human-to-human contact, high COVID-19 confirmation rates are observed in urbanized cities with high levels of air pollution. However, the role of air pollution and IPF in spreading COVID-19 infection is also not well understood. In the current study, we demonstrated that air pollution and IPF upregulate the protein levels of ACE2 and TMPRSS2 both in human tissue samples and animal studies, thereby providing a basis for their roles in spreading and exacerbating COVID-19 infection.

\section{Materials And Methods}

\section{Human lung tissue sections}

Human lung tissue sections were collected from patients of interstitial pneumonia who received lung resection surgery in 2000-2018. The Institutional Review Board of Taipei Veterans General Hospital approved the protocol and waived the need for informed consent.

\section{Animal models}


Eight-week-old male C57BL/6 mice were obtained from BioLASCO Co., Ltd. (Taipei, Taiwan) and

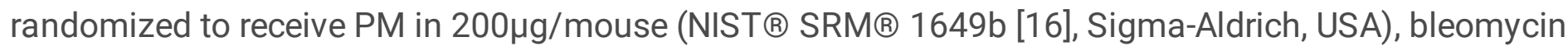
(Sigma-Aldrich, USA) in $2 \mathrm{U} / \mathrm{kg}$, bleomycin plus PM, or sterile PBS in contrl gruop via intratracheal administration. Animals were sacrificed on days 14 for further analyses. Reparixin was givene in 30 $\mathrm{mg} / \mathrm{kg}$ (Tocris, U.K.) by subcutaneous injection. All animal protocols were approve by the Institutional Animal Care and Use Committee of Taipei Veterans General Hospital.

\section{KC knockout mice}

KC knock out mice were generated using the CRISPR/Cas9 system, as described previously [17]. Briefly, Cas 9 mRNA and sgRNAs were microinjected into fertilized embryos of C57BL/6J mice. KC genotyping was performed by PCR with specific primers.

\section{Picro Sirius Red and Masson's trichrome stain}

Picro Sirius Red (Sigma-Aldrich, USA) and Masson's trichrome (Sigma-Aldrich, USA) were stained, ane the red color area (collagen) in the Picro Sirius Red image and the blue color in Masson's trichrome image were quantified using an image-analysis system (Image-Pro Plus; Media Cybernetics, USA).

\section{Immunohistochemistry and immunofluorescence}

After treatment with $3 \%$ hydrogen peroxide for 10 minutes, sections were blocked with $1 \%$ BSA, reacted with antibodies against ACE2 (Taiclone, Taiwan) or TMPRSS2 (Abcam, USA) for overnight. Sections were then incubated with peroxidase-labelled goat anti-mouse or goat anti-rabbit antibodies (Dako, USA). Peroxidase activity was developed by using 3,3'-diaminobenzidine chromogen (DAB) solution. Counterstained with haematoxylin, dehydrated, clean and mounted. Slides were imaged and quantified by microscopy (Nikon, USA). For immunofluorescence, sections were cincubated with fluorescenceconjugated $2^{\text {nd }}$ antibodies, followed by mounting with DAPI and imaged by fluorescence microscopy (Nikon, USA).

\section{Statistical analysis}

All statistical analyses were performed by Graphpad Prism 7.0. To compared the differrent groups, we used Kruskal-Wallis test and Dunn post hoc method considering nonparametric data. A value of $p<0.05$ was considered statistically significant. 


\section{Results}

\section{ACE2 and TMPRSS2 are present in normal human lung tissue}

To gain insight into the expression of ACE2 and TMPRSS2 in IPF and non-IPF control lung tissues, the immunostaining for both were mainly observed in alveolar cells in control lung tissues (Supplementary Figure 1), similar to the previous findings demonstrating the coexpression of ACE2 protein and TMPRSS2 in type II alveolar epithelial cells (pneumocytes) $[11,18]$. The human lung tissue sections of four IPF patients were first subjected to Picro Sirius Red and Masson's trichrome staining for histological confirmation of pulmonary fibrosis (Figure 1 and Supplementary Figure 2). This was the first study on ACE2 and TMPRSS2 immunostaining in patients with IPF. ACE2 and TMPRSS2 were both highly expressed in areas of pulmonary fibrosis (Figure 1). Similar data were observed in the other three IPF patients (Supplementary Figure 2). Collectively, these suggested that increased expression of ACE2 and TMPRSS2 was observed in pulmonary lung tissues of patients with IPF.

\section{Coexpression of ACE2 and TMPRSS2 in pulmonary fibroblasts of human lung fibrotic tissues}

IPF is characterized by progressive obliteration of normal alveolar architecture and replacement by fibrotic tissue [19]. Several studies have focused on determining the origin of fibroblasts and the signaling pathways leading to abnormal deposition of extracellular matrix by these fibroblasts. In addition, epithelial-mesenchymal transition (EMT) is a potential source of fibroblasts in lung tissue, with alveolar epithelial cells also acquiring the mesenchymal markers, such as fibroblast-specific protein 1 (FSP-1) $[20,21]$. We then investigated the kind of cells in human lung fibrotic tissues expressing ACE2 and TMPRSS2 through double immunofluorescence, which revealed co-expression of ACE2 and FSP-1 in human lung fibrotic tissue sections (Figure 2A). The co-expression of TMPRSS2 and FSP-1 was also observed in the lung sections (Figure 2B). These data suggested the expression of the coronavirus receptor ACE2 and the protease TMPRSS2 in FSP-1 positive fibroblasts in pulmonary lung tissues.

\section{PM upregulated ACE2 and TMPRSS2 expression}

EMT of both type II alveolar epithelial cells and bone marrow progenitors are the sites of origin of the fibroblasts in bleomycin-induced lung fibrosis [22]. Moreover, air pollution increases the severity of bleomycin-induced pulmonary fibrosis [17]. In the absence of a human model, we established a mouse model of bleomycin-induced lung fibrosis combined with administration of PM to investigate the effects of pulmonary fibrosis and air pollution on the expression of ACE2 andTMPRSS2. Mice receiving bleomycin with or without PM were sacrificed 14 days later, and lung tissues were collected for tissue sections. Masson's trichrome (Figure 3A) and Picro Sirius Red (Figure 3B) staining demonstrated successful induction of pulmonary fibrosis by bleomycin. Although administration of PM alone did not induce pulmonary fibrosis within 14 days, it significantly aggravated that caused by bleomycin (Figure 
3A, 3B, and 3D). Compared to the control group, mice receiving bleomycin with or without PM had increased expression of ACE2 and TMPRSS2 in lung tissue sections (Figure 3C). However, ACE2 and TMPPRSS2 were abundantly expressed and corresponded to lung fibrotic areas. Quantitative data further revealed that PM administration alone did not increase the expression of ACE2 and TMPRSS2 within 14 days, but it significantly increased collagen content and remarkably enhanced ACE2 and TMPRSS2 expression when combined with bleomycin treatment (Figure 3D). Similar to the human data, the positive staining of ACE2 (Supplementary Figure 3A) and TMPRSS2 (Supplementary Figure 3B) were both colocalized with the positive staining of FSP-1. Together, these data suggested that exposure to PM increased the severity of bleomycin-induced pulmonary fibrosis along with the upregulation of the levels of ACE2 and TMPRSS2.

\section{Keratinocyte chemoattractant (KC) depletion diminishes ACE2 and TMPRSS2 expression}

High concentrations of serum proteins, such as IL-8, predicted poor overall survival in IPF patients [23]. As previously reported, PM activates macrophages to secrete $\mathrm{KC}$, a murine homologue of IL-8, recruiting neutrophils and then increase the severity of bleomycin-induced IPF in mice [17]. These data suggest the important role of IL-8 in the develoment and severity of IPF. In addition, COVID-19 patients with chronic immune-mediated inflammatory diseases, such as IPF, are characterized by inherent immune dysfunction that leads to the release of cytokines, including IL-8 and disease severity [24, 25]. We therefore developed $\mathrm{KC}$ knockout mice ( $\mathrm{KC}^{-/}, \mathrm{KC}$-deficient) and treated them with bleomycin plus $\mathrm{PM}$ to explore the role of $\mathrm{KC}$ and the expression of ACE2 and TMPRSS2 in pulmonary fibrosis. Masson's trichrome (Figure 4A) and Picro Sirius Red (Figure 4B) staining revealed that KC depletion reduced the severity of pulmonary fibrosis. Moreover, the levels of ACE2 and TMPRSS2 were both reduced in KC-deficient mice in comparison with the control group (Figure 4C). Quantitative data revealed that KC depletion significantly reduced pulmonary fibrosis and the expression of ACE2 and TMPRSS2 (Figure 4D). These data suggested an important role of $\mathrm{KC}$ in PM-induced aggravation of pulmonary fibrosis and upregulation of ACE2 and TMPRSS2 in a bleomycin-induced pulmonary fibrosis model.

\section{Reparixin inhibited ACE2 and TMPRSS2 expression}

Reparixin, an inhibitor of IL-8 receptors CXCR1/2 [26], blocked PM-induced neutrophil accumulation and ameliorated the aggravation of pulmonary fibrosis induced by PM [17]. Masson's trichrome (Figure 5A) and Picro Sirius Red (Figure 5B) staining verified that reparixin ameliorated pulmonary fibrosis and reduced lung collagen contents. Co-treatment with reparixin in mice receiving PM and bleomycin also suppressed ACE2 and TMPRSS2 expression (Figure 5C). Quantitative data further showed that treatment with reparixin significantly improved pulmonary fibrosis and suppressed the levels of ACE2 and TMPRSS2 in mice receiving bleomycin plus PM (Figure 5D). These data indicated that treatment with reparixin improves lung fibrosis, and reduces the expression of ACE2 and TMPRSS2. 


\section{Discussion}

To the best of our knowledge, this is the first study to demonstrate the expression of COVID-19 receptor ACE2 and protease TMPRSS2 in patients with IPF through immunohistochemical methods. We have shown that ACE2 and TMPRSS2 are co-expressed in FSP-1 positive fibroblasts in human lung fibrotic tissues and in an animal lung fibrosis model. Importantly, PM upregulated ACE2 and TMPRSS2 protein levels, which were suppressed by $\mathrm{KC}$ deletion or treatment with reparixin, an inhibitor of chemokine receptors. These results provide insights into the worsening and risk factors of COVID-19 in patients with IPF.

COVID-19 is a viral disease with respiratory failure caused by SARS-CoV-2 entering cells through host ACE2 and TMPRSS2. ACE2, a functional receptor for SARS-CoV, is present in the lung alveolar epithelial cells [11]. We also found that alveolar lung cells were positive for ACE2 and TMPRSS2. Given that SARS-CoV-2 cell entry depends on host ACE2 and TMPRSS2 [12], the expression levels of ACE2 and TMPRSS2 may be assumed to affect transmissibility and susceptibility. Although the factors regulating ACE2 and TMPRSS2 are still unknown, both are co-expressed in lung type II pneumocytes as revealed by single-cell RNA-sequencing datasets [27].

These data indicated that alveolar lung cells in normal human lungs might be possible entry sites for SARS-CoV-2.

In light of the clinical manifestation of other comorbidities, ACE2 expression is elevated in the lungs of patients with morbidities correlated to severe COVID-19 [28]. Smoking was found to upregulate ACE2 expression [29]. Moreover, TMPRSS2 expression was found to be significantly elevated in smokers [30].The incremental expression of ACE2 and TMPRSS2 may explain the increased infection rate in smokers compared with nonsmokers [31]. However, the levels of ACE2 and TMPRSS2 mRNA are controversial in IPF patients. The mRNA level of ACE2 was increased in patients with IPF and interstitial lung disease (ILD) compared to controls, but that of TMPRSS2 did not show a difference in those with IPF [32]. There was no difference in gene expression of ACE2 and TMPRSS2 between IPF and healthy individuals, regardless of early and advanced IPF [30]. Collectively, previous studies showed stable levels of ACE2 and TMPRSS2 in patients with IPF.However, in our results, ACE2 and TMPRSS2 were expressed in lung tissue and co-localized with FSP-1 positive fibroblasts, and exhibited enhanced expression after the development of lung fibrosis. Although there are no reports on the incidence of SARS-CoV-2 infection in patients with IPF, such patients are extremely susceptible to COVID-19, when ACE2 and TMPRSS2 are co-expressed.

Air pollution, a major problem for the general population, is caused by a complex mixture of solid and liquid particles suspended in the air and various gases such as ozone $\left(\mathrm{O}_{3}\right), \mathrm{NO}_{2}$, and carbon monoxide (CO). Air pollutants can change host immunity to respiratory viral infections related to individual preexisting pulmonary conditions such as IPF, asthma, and chronic obstructive pulmonary disease (COPD) [33]. Johannsson et al. demonstrated an important relationship between the risk of acute exacerbation of IPF and $\mathrm{O}_{3}$ and $\mathrm{NO}_{2}$ [15]. IPF mortality was significantly associated with cumulative air 
pollution exposure to $\mathrm{PM}_{2.5}$ with a hazard ratio (HR) of 7.93 (95\% confidence interval (Cl) 2.93 to 21.33) and particulate matter $10\left(\mathrm{PM}_{10}\right)$ with a HR of 2.01 (95\% $\left.\mathrm{Cl} 1.07-3.77\right)$ [14]. The mechanisms of exposure to PM include EMT formation and fibronectin expression, and activation of transcription factors ETS-1 and NF-KB [34]. In addition, based on our findings, PM induced deterioration of pulmonary fibrosis in a bleomycin animal model and increased the expression of ACE2 and TMPRSS2. ACE2 expression in the mice lung increased at 2 and 5 days after $P M$ instillation, and $\mathrm{PM}_{2.5}$-induced acute lung injury in $A C E 2$ $\mathrm{KO}$ mice was more severe than that in the wild-type [35]. This could possibly be due to ACE2 having a protective role against acute lung injury [36]. Furthermore, TMPRSS2 enhances viral spread and pathogenesis by antibody-mediated neutralization and activation of virus-cell fusion [18]. Moreover, the formation of PM and viral microdroplets may also affect the severity of COVID-19 infection [37]. Based on these findings, we speculate that a combination of air pollution and pulmonary fibrosis will accelerate the spread and deterioration of COVID-19.

Although acute exacerbation of idiopathic pulmonary fibrosis (IPF-AE) is clinically important, the role of cytokines require further understanding. High interlukin-8 levels in the cytokine profile reflect lung inflammatory processes in IPF-AE patients [38]. The human IL-8 analog, KC, can aggravate inflammation and increase the levels of ACE2 and TMPRSS2. Moreover, as IPF progresses, fibrosis spreads widely in the alveolar walls, compromising alveoli function and gas exchange. However, the molecular mechanisms underlying fibrotic development have not been clarified. IL-8 mediates IPF mesenchymal progenitor cell fibrogenicity and prompts fibrotic progression by recruiting macrophages [39]. We further showed that the fibrosis status was reversed in KC-deficient mice compared with the wild-type when bleomycin and PM were received simultaneously. In the model of bleomycin-induced pulmonary fibrosis, treatment with reparixin improved the lung pathology and reduced collagen deposition. Reparixin, an inhibitor of IL-8 receptor such as CXCR1/2, inhibits neutrophil influx, fibrogenic cytokine, and decreases pulmonary fibrosis by blocking CXCR2 [40]. These data suggest that IL-8 and its downstream signaling induce lung function damage and upregulate ACE2 and TMPRSS2 expression. Furthermore, in IPF patients, Pirfenidone, a commercial antifibrotic drug with poor unknown mechanism, reduced disease progression, and increased lung function, exercise tolerance, and progression-free survival compared to placebo [41]. It has been suggested, there is no rationality to discontinue conventional antifibrotic therapy in patients with IPF who are infected with COVID-19 [8].

COVID-19 is an emerging pathogen, and modifying exposure of patients with IPF to air pollution is impossible. Our study is therefore subject to possible limitations, but human tissue and animal models were used in the study. Our results are mainly based on histological methods. Further studies are warranted to validate these results. Moreover, we failed to demonstrate the origins of FSP-1 positive fibroblasts co-expressing ACE2 and TMPRSS2 in human and murine IPF tissues and more susceptible to SARS-CoV-2 infection. Since previous studies demonstrated FSP-1 or a-smooth muscle actin (a-SMA) positive fibroblasts as derivatives from the lung residential alveolar epithetical cells, bone marrow mesenchymal cells [22], or endothelium [19], we cannot exclude any of these origins as the sources of ACE2 and TMPRSS2 expression in IPF lung tissues. 


\section{Conclusions}

Although the exact roles of ACE2 and TMPRSS2 as mediators of SARS-CoV-2 infection has not been well determined, the observed upregulation of both in air pollution, and pulmonary fibrosis may explain the spread and aggravation of COVID-19 infection by these two conditions. Further research proving the clinical significance of ACE2 and TMPRSS2 levels in COVID-19 infection and severity is required.

\section{Declarations}

\section{Acknowledgements}

We thank for Department of Pathology and Laboratory Medicine to assist for Masson's trichrome staining. We also thank for Medical Science \& Technology Building of Taipei Veterans General Hospital for experimental facilities. We thank Yu-Jr Lin in Research Services Center for Health Information, Chang Gung University for consulting all the statistics in this study.

\section{Authors' contribution}

H.-S. Hsu and S.-C. Hung conceived and designed research; H.-H. Li drafted manuscript; H.-S. Hsu and S.C. Hung edited and revised manuscript. H.-H. Li, J.-H. Lin, T.-W. Hsu, J.-W. Hsu, Y.-C. Yeh and A. F.-Y. Li. performed experiments and analyzed data. C.-C. Liu, Y.-C. Yeh, A. F.-Y. Li. studied the biological plausibility of findings and interpreted the results from a clinical point of view. H.-H. Li and T.-W. Hsu prepared figures. All authors interpreted results of experiments and approved the final version of manuscript before the submission.

\section{Funding}

This work was financially supported by Ministry of Science and Technology (MOST 106-2321-B-039 -003; 109-2321-B-039-003).

\section{Availability of data and materials}

All the data and materials presented in the study with additional files are available.

\section{Ethics approval and consent to participate}

The Institutional Review Board of Taipei Veterans General Hospital approved the protocol and waived the need for informed consent (IRB approval number: 2020-09-016CC). All animal protocols were approved 
by the Institutional Animal Care and Use Committee of Taipei Veterans General Hospital (IACUC approval number: 2017-003).

\section{Consent for publication}

Not applicable.

\section{Competing interests}

All of the authors declare that there is no conflict of interest.

\section{References}

1. Zhu Y, Xie J, Huang F, Cao L. Association between short-term exposure to air pollution and COVID-19 infection: Evidence from China. Sci Total Environ. 2020;727:138704.

2. Zoran MA, Savastru RS, Savastru DM, Tautan MN. Assessing the relationship between surface levels of PM2.5 and PM10 particulate matter impact on COVID-19 in Milan, Italy. Sci Total Environ. 2020;738:139825.

3. Cui Y, Zhang ZF, Froines J, Zhao J, Wang H, Yu SZ, et al. Air pollution and case fatality of SARS in the People's Republic of China: an ecologic study. Environ Health. 2003;2 1:15.

4. Conticini E, Frediani B, Caro D. Can atmospheric pollution be considered a co-factor in extremely high level of SARS-CoV-2 lethality in Northern Italy? Environ Pollut. 2020;261:114465.

5. Setti L, Passarini F, Gennaro Gd, Gilio AD, Palmisani J, Buono P, et al. Evaluation of the potential relationship between Particulate Matter (PM)pollution and COVID-19 infection spread in Italy. Italian Society of Environmental Medicine. 2020.

6. Raghu G, Collard HR, Egan JJ, Martinez FJ, Behr J, Brown KK, et al. An official ATS/ERS/JRS/ALAT statement: idiopathic pulmonary fibrosis: evidence-based guidelines for diagnosis and management. Am J Respir Crit Care Med. 2011;183 6:788-824.

7. King CS, Nathan SD. Idiopathic pulmonary fibrosis: effects and optimal management of comorbidities. Lancet Respir Med. 2017;5 1:72-84.

8. George PM, Wells AU, Jenkins RG. Pulmonary fibrosis and COVID-19: the potential role for antifibrotic therapy. Lancet Respir Med. 2020.

9. Drake TM, Docherty AB, Harrison EM, Quint JK, Adamali H, Agnew S, et al. Outcome of Hospitalization for COVID-19 in Patients with Interstitial Lung Disease: An International Multicenter Study. Am J Respir Crit Care Med. 2020.

10. Mora AL, Rojas M, Pardo A, Selman M. Emerging therapies for idiopathic pulmonary fibrosis, a progressive age-related disease. Nat Rev Drug Discov. 2017;16 11:810. 
https://www.ncbi.nlm.nih.gov/pubmed/29081515.

11. Hamming I, Timens W, Bulthuis ML, Lely AT, Navis G, van Goor H. Tissue distribution of ACE2 protein, the functional receptor for SARS coronavirus. A first step in understanding SARS pathogenesis. $J$ Pathol. 2004;203 2:631-7.

12. Hoffmann M, Kleine-Weber H, Schroeder S, Krüger N, Herrler T, Erichsen S, et al. SARS-CoV-2 Cell Entry Depends on ACE2 and TMPRSS2 and Is Blocked by a Clinically Proven Protease Inhibitor. Cell. 2020;181 2:271-80.e8.

13. Conti S, Harari S, Caminati A, Zanobetti A, Schwartz JD, Bertazzi PA, et al. The association between air pollution and the incidence of idiopathic pulmonary fibrosis in Northern Italy. Eur Respir $\mathrm{J}$. 2018;51 1. https://www.ncbi.nIm.nih.gov/pubmed/29371377.

14. Sesé L, Nunes H, Cottin V, Sanyal S, Didier M, Carton Z, et al. Role of atmospheric pollution on the natural history of idiopathic pulmonary fibrosis. Thorax. 2018;73 2:145-50.

15. Johannson KA, Vittinghoff E, Lee K, Balmes JR, Ji W, Kaplan GG, et al. Acute exacerbation of idiopathic pulmonary fibrosis associated with air pollution exposure. Eur Respir J. 2014;43 4:112431.

16. Albinet A, Lanzafame GM, Srivastava D, Bonnaire N, Nalin F, Wise SA. Analysis and determination of secondary organic aerosol (SOA) tracers (markers) in particulate matter standard reference material (SRM 1649b, urban dust). Anal Bioanal Chem. 2019;411 23:5975-83. https://www.ncbi.nlm.nih.gov/pubmed/31312884.

17. Cheng IY, Liu CC, Lin JH, Hsu TW, Hsu JW, Li AF, et al. Particulate Matter Increases the Severity of Bleomycin-Induced Pulmonary Fibrosis through KC-Mediated Neutrophil Chemotaxis. Int J Mol Sci. 2019;21 1.

18. Glowacka I, Bertram S, Müller MA, Allen P, Soilleux E, Pfefferle S, et al. Evidence that TMPRSS2 activates the severe acute respiratory syndrome coronavirus spike protein for membrane fusion and reduces viral control by the humoral immune response. J Virol. 2011;85 9:4122-34.

19. Nataraj D, Ernst A, Kalluri R. Idiopathic pulmonary fibrosis is associated with endothelial to mesenchymal transition. Am J Respir Cell Mol Biol. 2010;43 2:129-30. https://www.ncbi.nlm.nih.gov/pubmed/20651063.

20. Kim KK, Kugler MC, Wolters PJ, Robillard L, Galvez MG, Brumwell AN, et al. Alveolar epithelial cell mesenchymal transition develops in vivo during pulmonary fibrosis and is regulated by the extracellular matrix. Proc Natl Acad Sci U S A. 2006;103 35:13180-5.

21. Willis BC, Liebler JM, Luby-Phelps K, Nicholson AG, Crandall ED, du Bois RM, et al. Induction of epithelial-mesenchymal transition in alveolar epithelial cells by transforming growth factor-beta1: potential role in idiopathic pulmonary fibrosis. Am J Pathol. 2005;166 5:1321-32. https://www.ncbi.nlm.nih.gov/pubmed/15855634.

22. Tanjore H, Xu XC, Polosukhin VV, Degryse AL, Li B, Han W, et al. Contribution of epithelial-derived fibroblasts to bleomycin-induced lung fibrosis. Am J Respir Crit Care Med. 2009;180 7:657-65. https://www.ncbi.nlm.nih.gov/pubmed/19556518. 
23. Richards TJ, Kaminski N, Baribaud F, Flavin S, Brodmerkel C, Horowitz D, et al. Peripheral blood proteins predict mortality in idiopathic pulmonary fibrosis. Am J Respir Crit Care Med. 2012;185 1:6776. https://www.ncbi.nlm.nih.gov/pubmed/22016448.

24. Moore JB, June CH. Cytokine release syndrome in severe COVID-19. Science. 2020;368 6490:473-4. https://www.ncbi.nlm.nih.gov/pubmed/32303591.

25. Schett G, Sticherling M, Neurath MF. COVID-19: risk for cytokine targeting in chronic inflammatory diseases? Nat Rev Immunol. 2020;20 5:271-2. https://www.ncbi.nlm.nih.gov/pubmed/32296135.

26. Moriconi A, Cesta MC, Cervellera MN, Aramini A, Coniglio S, Colagioia S, et al. Design of noncompetitive interleukin-8 inhibitors acting on CXCR1 and CXCR2. J Med Chem. 2007;50 17:39844002. https://www.ncbi.nlm.nih.gov/pubmed/17665889.

27. Ziegler CGK, Allon SJ, Nyquist SK, Mbano IM, Miao VN, Tzouanas CN, et al. SARS-CoV-2 Receptor ACE2 Is an Interferon-Stimulated Gene in Human Airway Epithelial Cells and Is Detected in Specific Cell Subsets across Tissues. Cell. 2020;181 5:1016-35.e19.

28. Pinto BGG, Oliveira AER, Singh Y, Jimenez L, Gonçalves ANA, Ogava RLT, et al. ACE2 Expression is Increased in the Lungs of Patients with Comorbidities Associated with Severe COVID-19. medRxiv. 2020.

29. Smith JC, Sausville EL, Girish V, Yuan ML, Vasudevan A, John KM, et al. Cigarette Smoke Exposure and Inflammatory Signaling Increase the Expression of the SARS-CoV-2 Receptor ACE2 in the Respiratory Tract. Dev Cell. 2020;53 5:514-29.e3.

30. Saheb Sharif-Askari N, Saheb Sharif-Askari F, Alabed M, Temsah MH, Al Heialy S, Hamid Q, et al. Airways Expression of SARS-CoV-2 Receptor, ACE2, and TMPRSS2 Is Lower in Children Than Adults and Increases with Smoking and COPD. Mol Ther Methods Clin Dev. 2020;18:1-6.

31. Cai H. Sex difference and smoking predisposition in patients with COVID-19. Lancet Respir Med. 2020;8 4:e20.

32. Xu J, Xu X, Jiang L, Dua K, Hansbro PM, Liu G. SARS-CoV-2 induces transcriptional signatures in human lung epithelial cells that promote lung fibrosis. Respir Res. 2020;21 1:182.

33. Ciencewicki J, Jaspers I. Air pollution and respiratory viral infection. Inhal Toxicol. 2007;19 14:113546.

34. Chen YC, Chuang TY, Liu CW, Liu CW, Lee TL, Lai TC, et al. Particulate matters increase epithelialmesenchymal transition and lung fibrosis through the ETS-1/NF-KB-dependent pathway in lung epithelial cells. Part Fibre Toxicol. 2020;17 1:41.

35. Lin Cl, Tsai CH, Sun YL, Hsieh WY, Lin YC, Chen CY, et al. Instillation of particulate matter 2.5 induced acute lung injury and attenuated the injury recovery in ACE2 knockout mice. Int J Biol Sci. 2018;14 3:253-65.

36. Imai Y, Kuba K, Rao S, Huan Y, Guo F, Guan B, et al. Angiotensin-converting enzyme 2 protects from severe acute lung failure. Nature. 2005;436 7047:112-6.

37. Fronza R, Lusic M, Schmidt M, Lucic B. Spatial-Temporal Variations in Atmospheric Factors Contribute to SARS-CoV-2 Outbreak. Viruses. 2020;12 6. 
38. Papiris SA, Tomos IP, Karakatsani A, Spathis A, Korbila I, Analitis A, et al. High levels of IL-6 and IL-8 characterize early-on idiopathic pulmonary fibrosis acute exacerbations. Cytokine. 2018;102:168-72.

39. Yang L, Herrera J, Gilbertsen A, Xia H, Smith K, Benyumov A, et al. IL-8 mediates idiopathic pulmonary fibrosis mesenchymal progenitor cell fibrogenicity. Am J Physiol Lung Cell Mol Physiol. 2018;314 1:L127-I36.

40. Russo RC, Guabiraba R, Garcia CC, Barcelos LS, Roffê E, Souza AL, et al. Role of the chemokine receptor CXCR2 in bleomycin-induced pulmonary inflammation and fibrosis. Am J Respir Cell Mol Biol. 2009;40 4:410-21.

41. King TE, Jr., Bradford WZ, Castro-Bernardini S, Fagan EA, Glaspole I, Glassberg MK, et al. A phase 3 trial of pirfenidone in patients with idiopathic pulmonary fibrosis. N Engl J Med. 2014;370 22:208392.

\section{Figures}
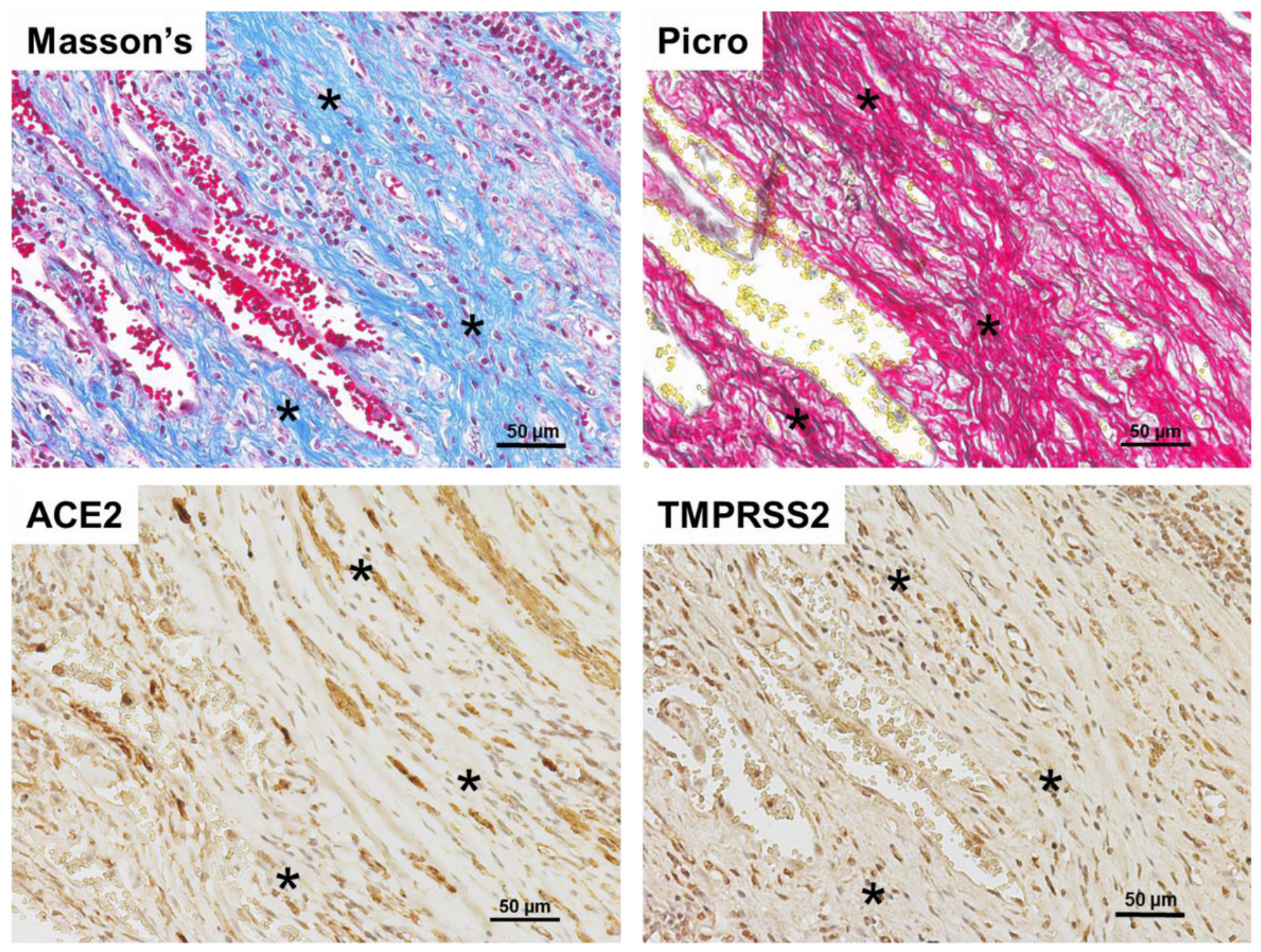

\section{Figure 1}


Expression of ACE2 and TMPRSS2 in pulmonary fibrosis tissue. Masson's trichrome, Picro Sirius Red staining and immunohistochemistry for ACE2 and TMPRSS2 were performed in continuous lung tissue sections from IPF patients. The fibrotic areas, stained as blue in Masson's trichrome or red in Picro Sirius Red, express strong ACE2 and TMPRSS2 staining (stars). Scale bar: $50 \mu \mathrm{m}$. ACE2: angiotensin-converting enzyme 2; TMPRSS2: transmembrane serine protease 2; IPF: idiopathic pulmonary fibrosis.

(A)
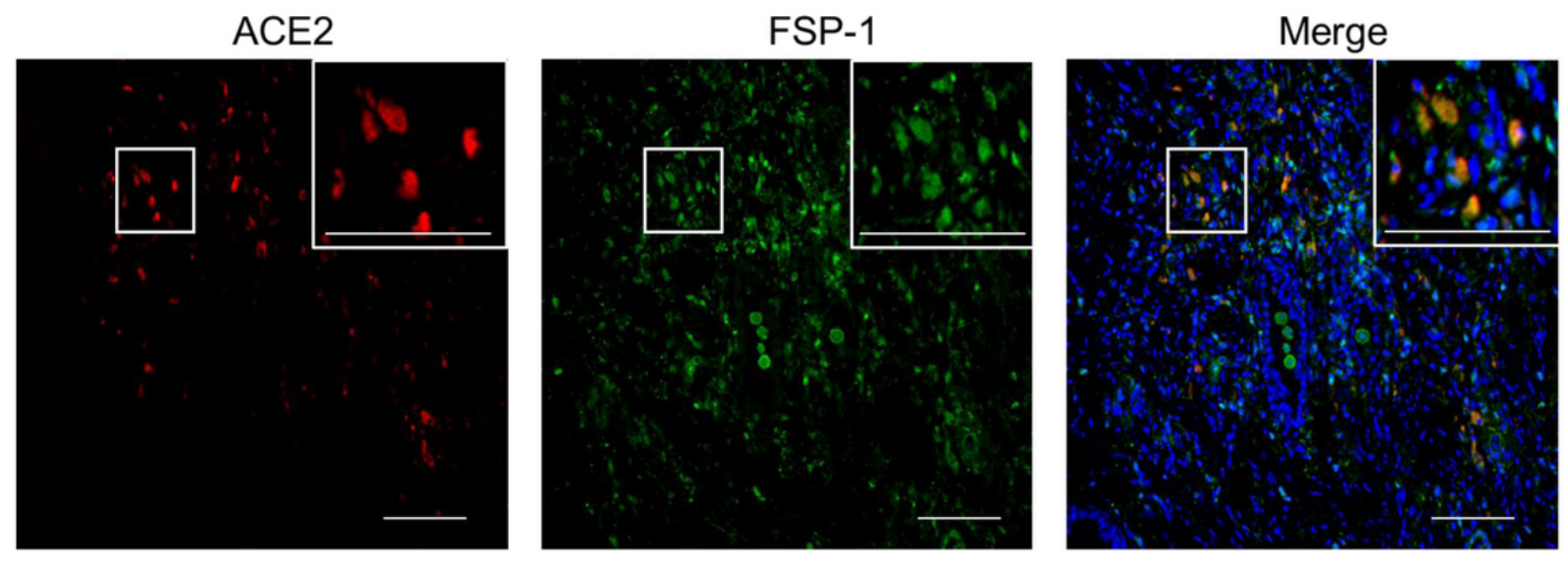

(B)

TMPRSS2

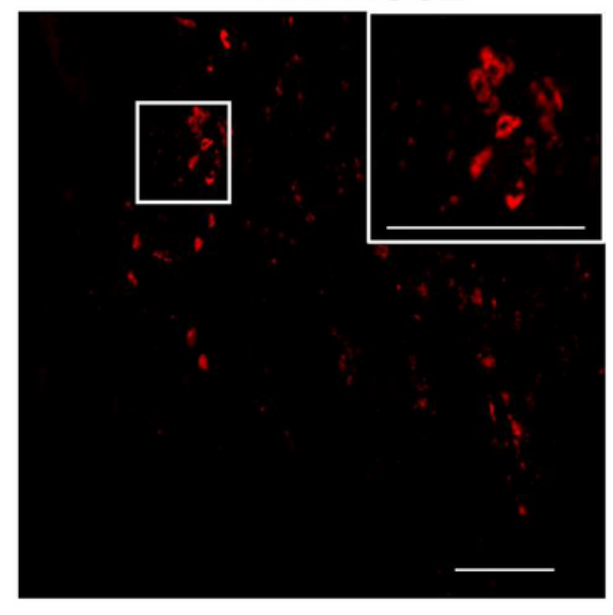

FSP-1

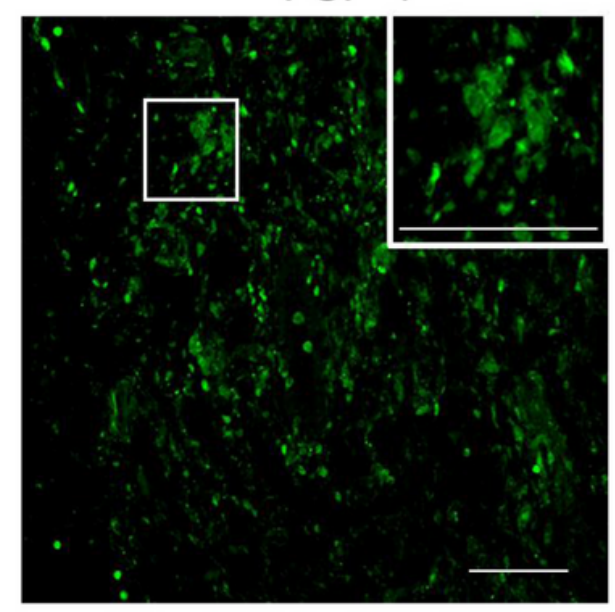

Merge

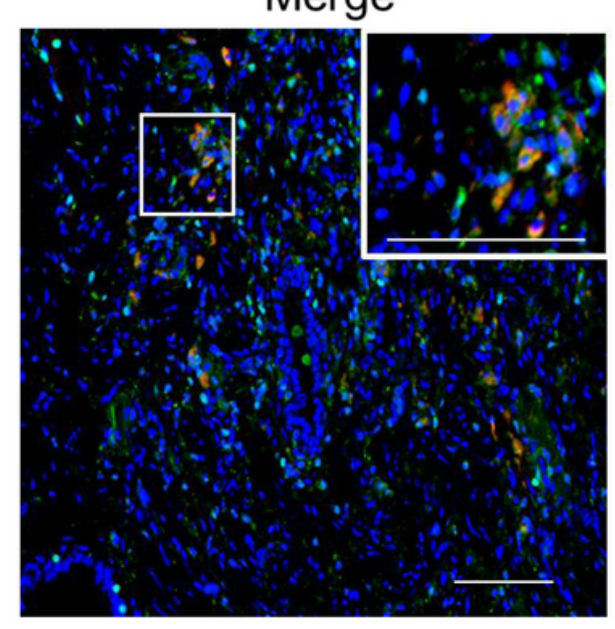

Figure 2

Expression of ACE2 and TMPRSS2 in FSP-1 positive pulmonary fibrosis areas. Double immunofluorescence was performed in continuous lung tissue sections from IPF patients. Representative images showing the colocalization of (A) ACE2 or (B) TMPRSS2 with fibroblast marker, FSP-1. The rectangle frames are magnified on the right upper corners. Scale bar: $100 \mu \mathrm{m}$. ACE2: angiotensinconverting enzyme 2; TMPRSS2: transmembrane serine protease 2; FSP-1: fibroblast-specific protein 1; IPF: idiopathic pulmonary fibrosis. 
(A)

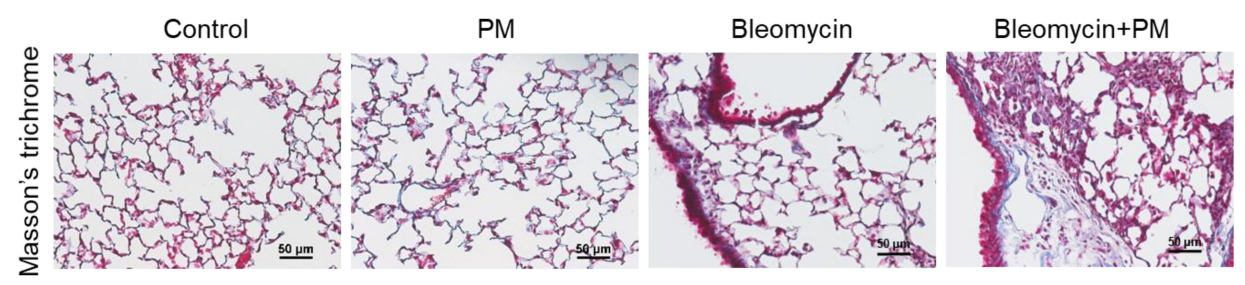

(B)

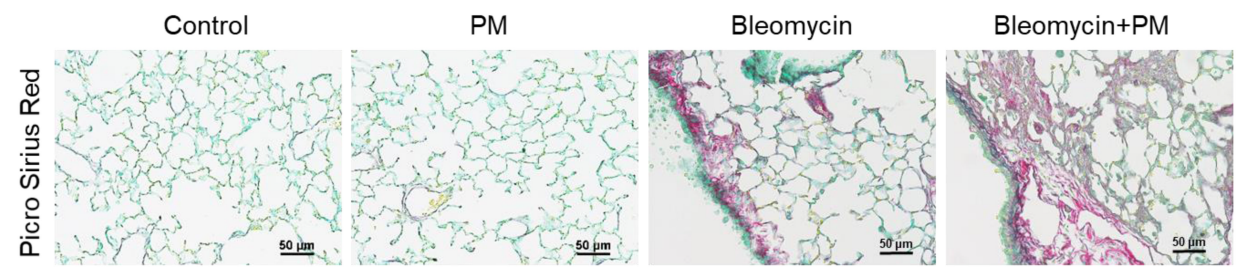

(C)

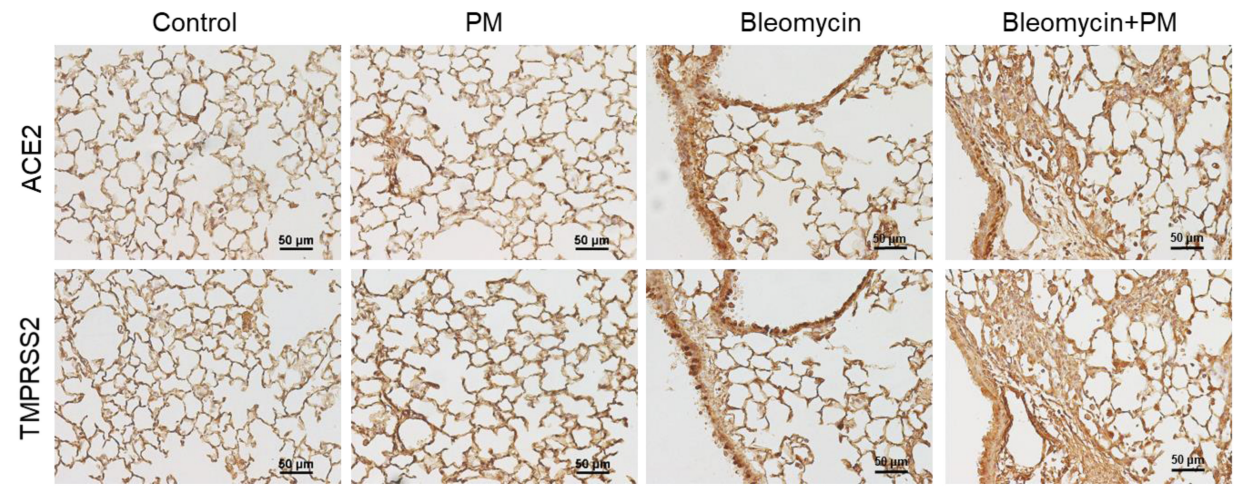

(D)

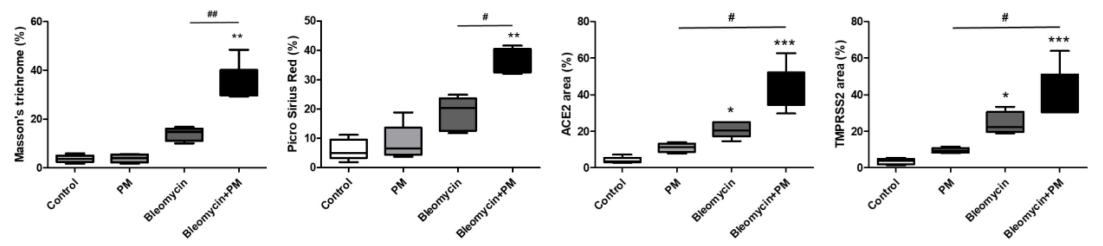

\section{Figure 3}

Mice lung tissue sections from different groups were stained by immunohistochemistry, Masson's trichrome and Picro Sirius Red. (A) The blue color in Masson's trichrome staining indicates collagen fiber, while (B) red color in Picro Sirius Red staining indicates collagen. These data demonstrate that PM aggravated bleomycin-induced pulmonary fibrosis. (C) Elevated levels of ACE2 and TMPRSS2 are observed in the bleomycin and PM group. These data indicate that PM upregulates the expression of 
ACE2 and TMPRSS2, mainly in areas with exacerbation of pulmonary fibrosis. (D) In the boxplots, quantification of pulmonary fibrosis and the levels of ACE2 and TMPRSS2. Data are shown as the median value with the interquartile range. Scale bar: $50 \mathrm{Im}$. P values were determined by Kruskal-Wallis test as ${ }^{*} p<0.05, * * p<0.001$ and ${ }^{* * *} p<0.001$ compared with control. $\# p<0.05$ and $\# \# p<0.01$ compared with bleomycin plus PM group. PM: particulate matter; ACE2: angiotensin-converting enzyme 2; TMPRSS2: transmembrane serine protease 2. ( $n=4$ per group)

(A)
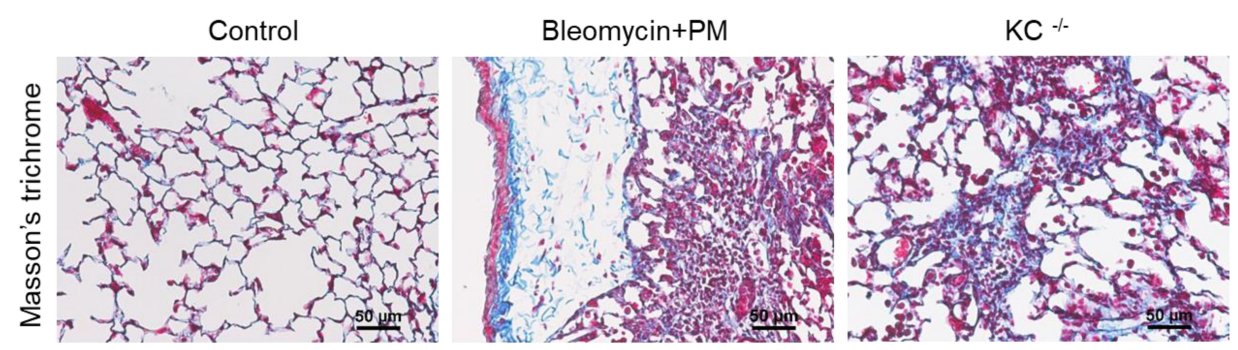

(B)
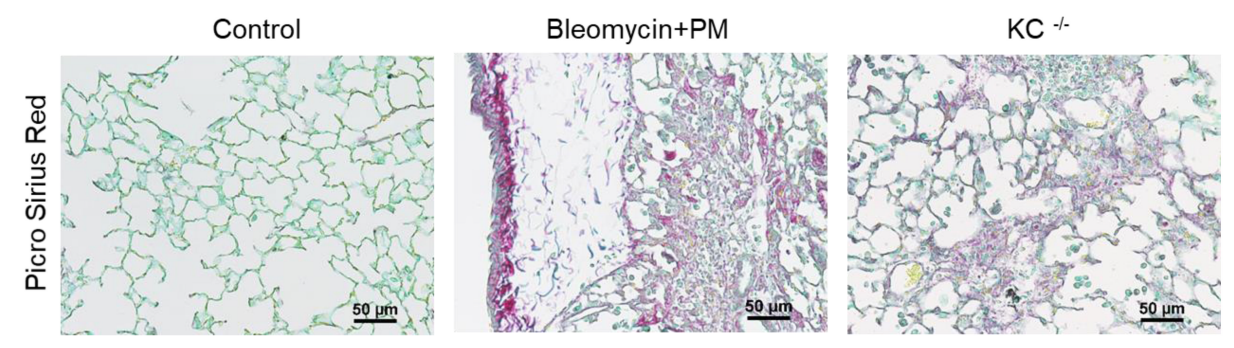

(C)
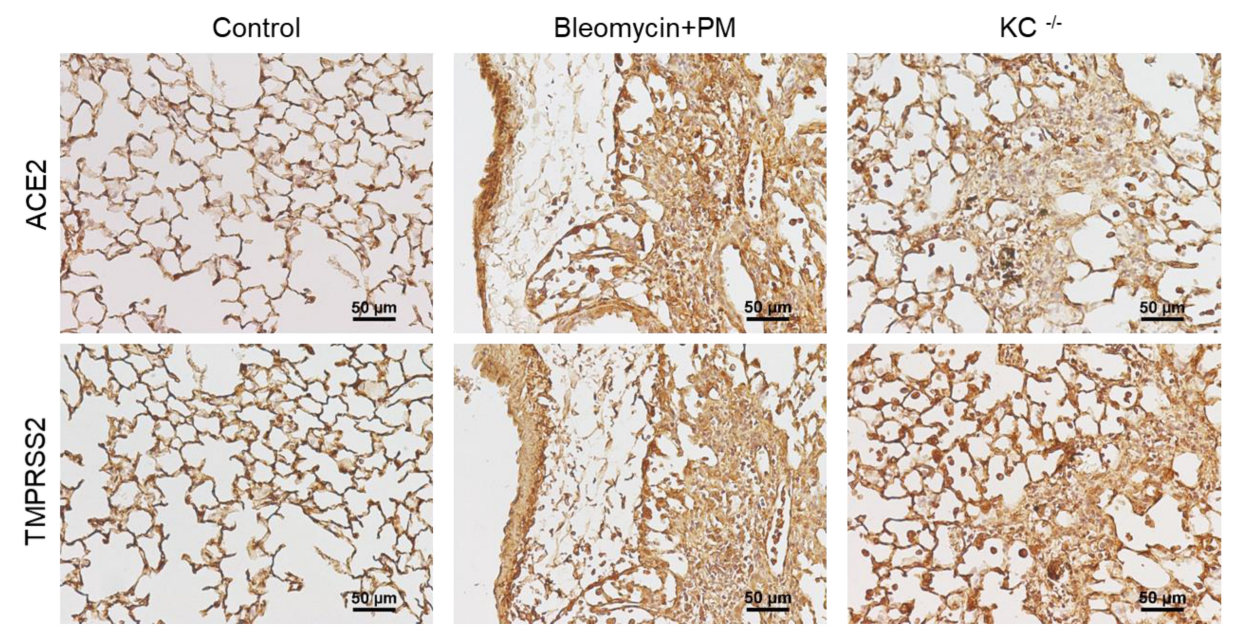

(D)

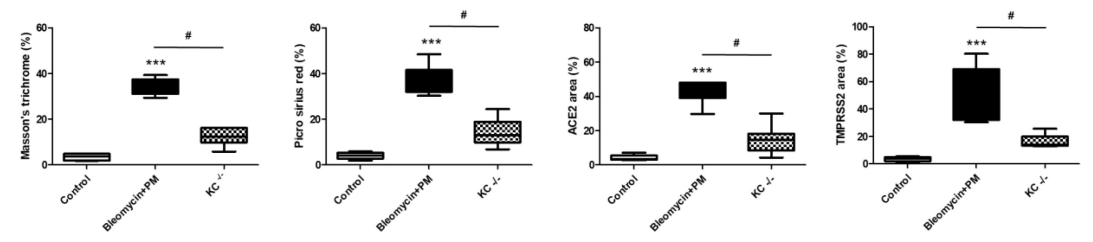


KC diminished pulmonary fibrosis development and ACE2 and TMPRSS2 exhibition. (A) Positive Masson's trichrome staining and (B) Picro Sirius Red staining symbolize pulmonary fibrosis. (C) Immunohistochemistry for ACE2 and TMPRSS2. Pulmonary fibrosis and the expression of ACE2 and TMPRSS2 declined in KC knockout mice. (D) Quantification of pulmonary fibrosis and ACE2 and TMPRSS2 expression. Data are shown as the median value with the interquartile range. Scale bar: $50 \mathrm{Im}$. $P$ values were determined by Kruskal-Wallis test as ${ }^{\star \star *} p<0.001$ compared with control group. $\# p<0.05$ compared with bleomycin plus PM group. KC: keratinocyte chemoattractant; ACE2: angiotensinconverting enzyme 2; TMPRSS2: transmembrane serine protease 2; PM: particulate matter. $(n=4$ per group) 
(A)

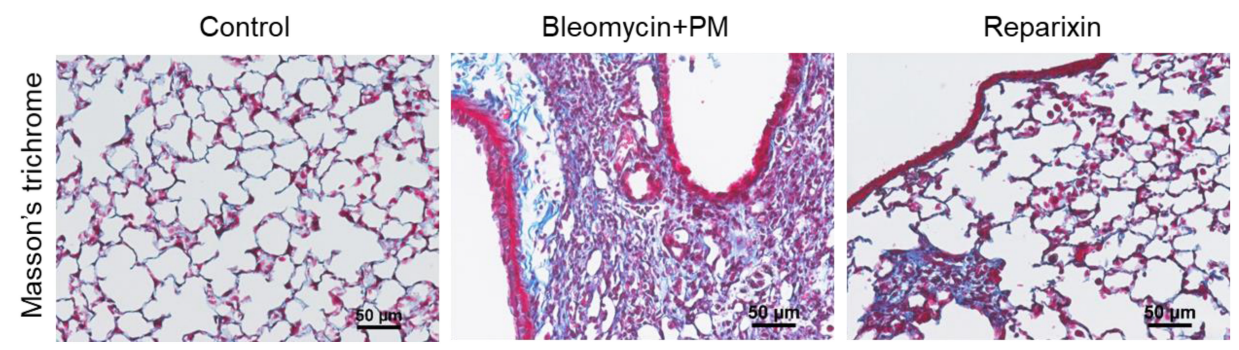

(B)

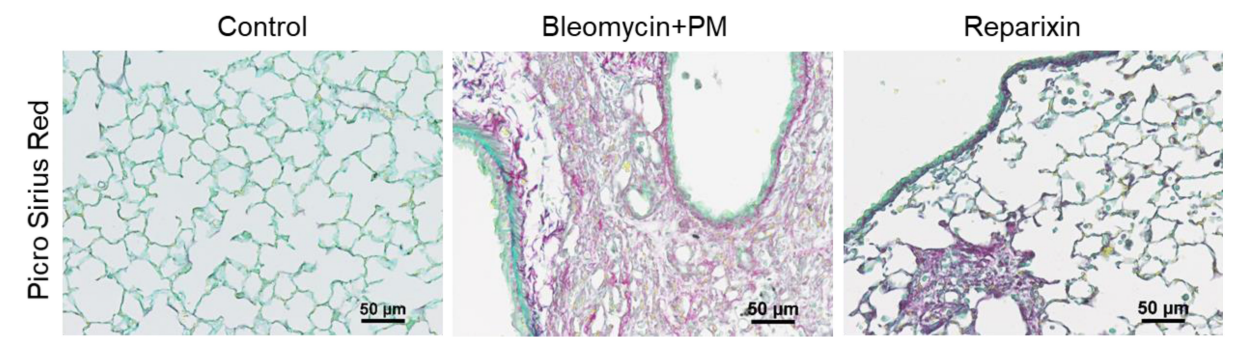

(C)

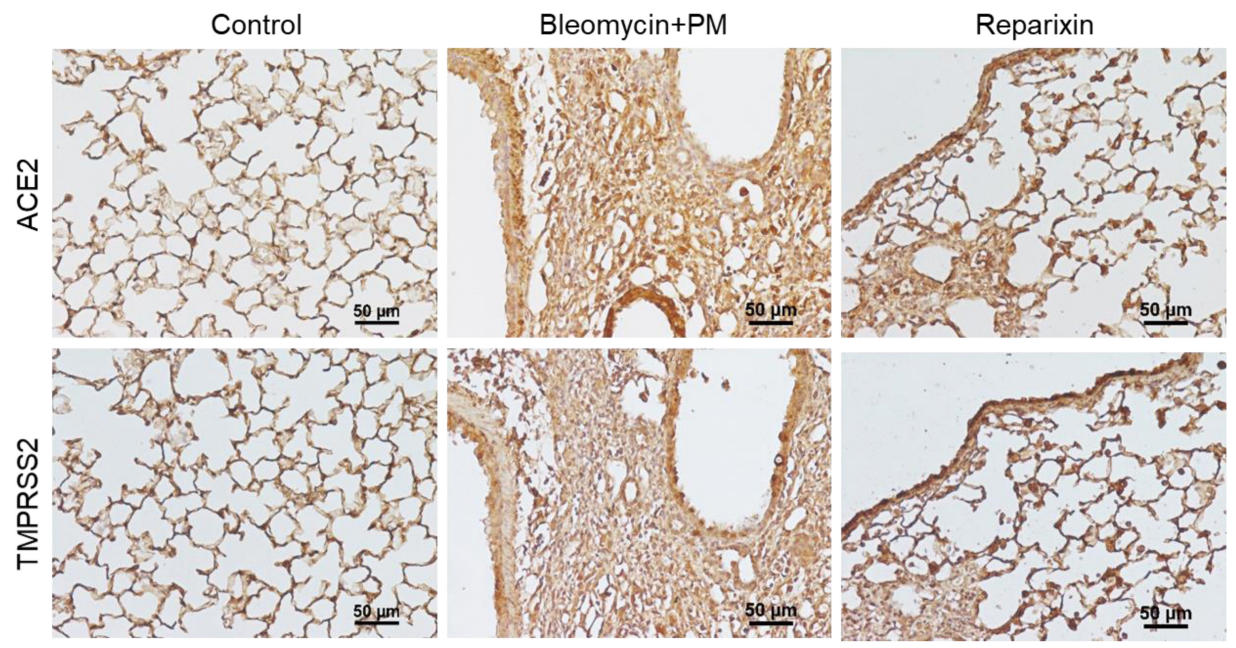

(D)

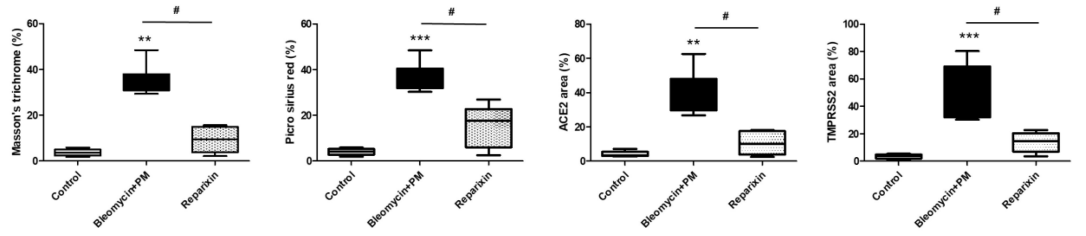

\section{Figure 5}

Treatment with Reparixin ameliorated PM-enhanced pulmonary fibrosis and diminished ACE2 and TMPRSS2 expression. (A) Positivities in Masson's trichrome staining and (B) Picro Sirus Red staining symbolize pulmonary fibrosis. (C) Immunohistochemistry for ACE2 and TMPRSS2. Pulmonary fibrosis and the expression of ACE2 and TMPRSS2 declined with Reparixin treatment. (D) All boxpolts show the quantification of pulmonary fibrosis and ACE2 and TMPRSS2 expression. Data are shown as the median 
value with the interquartile range. Scale bar: $50 \mu \mathrm{m}$. P values were determined by Kruskal-Wallis test as ** $\mathrm{p}<0.01$ and ${ }^{* \star *} \mathrm{p}<0.001$ and compared with control group. $\# \mathrm{p}<0.05$ compared with bleomycin plus PM group. PM: particulate matter; ACE2: angiotensin-converting enzyme 2; TMPRSS2: transmembrane serine protease 2 . $(n=4$ per group)

\section{Supplementary Files}

This is a list of supplementary files associated with this preprint. Click to download.

- SupplementalFigure.pdf 